\title{
UNIQUE FACTORISATION RINGS
}

\author{
by A. W. CHATTERS, M. P. GILCHRIST and D. WILSON
}

(Received 22nd June 1990)

\begin{abstract}
Let $R$ be a ring. An element $p$ of $R$ is a prime element if $p R=R p$ is a prime ideal of $R$. A prime ring $R$ is said to be a Unique Factorisation Ring if every non-zero prime ideal contains a prime element. This paper develops the basic theory of U.F.R.s. We show that every polynomial extension in central indeterminates of a U.F.R. is a U.F.R. We consider in more detail the case when a U.F.R. is either Noetherian or satisfies a polynomial identity. In particular we show that such a ring $R$ is a maximal order, that every height-1 prime ideal of $R$ has a classical localisation in which every two-sided ideal is principal, and that $R$ is the intersection of a left and right Noetherian ring and a simple ring.
\end{abstract}

1980 Mathematics subject classification (1985 Revision): 16A02, 16A38.

\section{Introduction}

The concept of a unique factorisation domain in commutative algebra was extended in two different ways to non-commutative rings in [5] and [6]. However, these generalisations were only studied in the context of Noetherian rings. Even here there is a surprisingly rich supply of genuinely non-commutative examples, including trace rings of generic matrix rings and many twisted polynomial rings, group rings and universal enveloping algebras (see [1], [5], [6], [13]). The aim of this paper is to develop a theory of non-commutative U.F.R.s (unique factorisation rings) without the Noetherian condition. The non-Noetherian setting is perhaps the right one in which to study U.F.R.s, but dropping the Noetherian condition does introduce extra technical problems. The precise definition of a U.F.R. is given in Section 2 and some general results are proved in Section 3. For example, if $R$ is a U.F.R. then so also is the ring of polynomials over $R$ in an arbitrary number of central indeterminates (Theorem 3.7). It is also possible to prove a non-Noetherian version of the theorem of Gilchrist and Smith, that certain U.F.R.s which are not commutative are principal ideal domains (Theorem 3.8).

After a certain point it is difficult to extend the general theory any further without being able to use Goldie's theorem. For this reason we specialise in Section 4 to U.F.R.S which satisfy a polynomial identity, because one feature of such rings is that all their prime factor rings are Goldie rings. For these rings the theory can be pushed much further. Such a ring $R$ satisfies the standard non-commutative version of being integrally-closed (Corollary 4.8), the height-1 prime ideals of $R$ are localisable (Theorem 4.6), the invertible ideals of $R$ are principal (Corollary 4.17), and $R$ is the intersection of 
its simple Artinian quotient ring with a left and right Noetherian ring in which every two-sided ideal is principal (Theorem 4.13).

\section{Definitions, notation and preliminaries}

All rings considered here are associative with identity element. Let $R$ be a ring. An element $x$ of $R$ is normal if $x R=R x$. Normal elements $x$ and $y$ of $R$ are associates if $x R=y R$. A principal ideal of $R$ is an ideal of the form $x R$ for some normal element $x$ of $R$. An element is regular if it is not a zero-divisor. If $I$ is an ideal of $R$ then $C(I)$ denotes the set of elements of $R$ which are regular modulo $I$.

General background material about ring theory can be found in [4] or [15]. For the theory of maximal orders we refer to [14], and for rings satisfying polynomial identities we refer to [16].

Let $R$ be a prime ring. A prime element of $R$ is a non-zero normal element $p$ such that $p R$ is a prime ideal. Such an element $p$ is said to be completely prime if $R / p R$ is an integral domain. We say that $R$ is a U.F.R. (unique factorisation ring) if every non-zero prime ideal of $R$ contains a prime element, and that $R$ is a U.F.D. (unique factorisation domain) if $R$ is an integral domain and every non-zero prime ideal contains a completely prime element. These definitions were introduced for Noetherian rings in [5] and [6].

Notation 2.1. Let $R$ be a U.F.R. Set

$$
C=\{c \in R: c \in C(p R) \text { for every prime element } p \text { of } R\} .
$$

We shall repeatedly use the fact that if $x$ is a normal element of $R$ and $P$ is a prime ideal of $R$, then either $x \in P$ or $x \in C(P)$; this is because $(x R+P) / P=(R x+P) / P$ is a twosided ideal of the prime ring $R / P$, and an ideal of a prime ring is either 0 or its left and right annihilators are 0 .

\section{General theory}

In this section we establish some basic properties of U.F.R.s without making any extra assumptions such as chain conditions. In particular we shall show that if $R$ is a U.F.R. then the set of principal ideals of $R$ is closed under finite intersections and satisfies the ascending chain condition (Theorem 3.5), and the polynomial ring over $R$ in an arbitrary number of central indeterminates is also a U.F.R. (Theorem 3.7). Provided that things are done in the correct order, the proofs are usually not hard and we shall keep them as short as possible. The results of this section are what one might reasonably hope for in view of the commutative theory of U.F.D.'s (see for example [12]).

Lemma 3.1. Let $p$ and $q$ be prime elements of a prime ring $R$. 
(1) If $q R \subseteq p R$ then $q R=p R$.

(2) If $p R \neq q R$ then $p q R=p R \cap q R=q p R$,

Proof. (1) Suppose that $q R \subseteq p R$. Then $q=p x$ for some $x \in R$. Suppose that $q R \neq p R$. Then $p \in C(q R)$. Because $p x \in q R$, we conclude that $x=y q$ for some $y \in R$. Hence $q=p y q$ and $1=p y$. This is a contradiction because $p R \neq R$.

(2) Suppose that $p R \neq q R$. Then $p \in C(q R)$ and $q \in C(p R)$. Let $x \in p R \cap q R$. Then $x=p y=q z$ for some $y, z \in R$. Because $p \in C(q R), y=q r$ for some $r \in R$. Thus $x=p q r$. It follows that $p R \cap q R \subseteq p q R$, and the rest of the proof is straightforward.

Lemma 3.2. Let $R$ be a U.F.R.

(1) Every non-zero ideal of $R$ contains a product of prime elements.

(2) Let $x$ be a non-zero element of $R$. Then there are only finitely-many non-associate prime elements $p$ of $R$ such that $x \in p R$.

Proof. (1) Apply Zorn's lemma to the set of ideals of $R$ which do not contain a product of prime elements.

(2) Let $x$ be a non-zero element of $R$. By (1) there are prime elements $p_{1}, \ldots, p_{n}$ of $R$ such that $p_{1} p_{2} \ldots p_{n} \in R \times R$. Let $p$ be a prime element of $R$ such that $x \in p R$. We have $p_{1} p_{2} \ldots p_{n} \in p R$ where each $p_{i}$ is a normal element of $R$. Hence $p_{i} \in p R$ for some $i$. Thus we have $p R=p_{i} R$ for some $i$, by Lemma 3.1, and so there are only finitely-many possibilities for $p R$.

Theorem 3.3. Let $R$ be a U.F.R. and let $p$ be a prime element of $R$.

(1) The prime ideal $p R$ has height 1 .

(2) $\bigcap_{n=1}^{\infty} p^{n} R=0$.

(3) $C(p R) \subseteq C\left(p^{n} R\right)$ for every positive integer $n$.

(4) The elements of $C(p R)$ are regular as elements of $R$.

(5) Let $x$ be a normal element of $R$ with $x \notin p R$. Then $x R \cap p^{n} \dot{R}=x p^{n} R$ for every positive integer $n$.

Proof. (1) Suppose that $Q$ is a non-zero prime ideal of $R$ with $Q \subseteq p R$. We must show that $Q=p R$. Because $R$ is a U.F.R., there is a prime element $q$ of $R$ such that $q \in Q$. Hence $q R \subseteq p R$. By Lemma 3.1 we have $q R=p R$ so that $Q=p R$.

(2) Set $I=\bigcap_{n=1}^{\infty} p^{n} R$ and suppose that $I \neq 0$. Because $p$ is a regular element of $R$ it is easy to show that $I=p I$. By Lemma 3.2 there are prime elements $p_{1}, \ldots, p_{n}$ of $R$ such that $p_{1} p_{2} \ldots p_{n} \in I$. Let $n$ be the smallest positive integer such that $I$ contains such a product. Then $p_{1} p_{2} \ldots p_{n} \in p R$ so that $p_{i} \in p R$ for some $i$. By 3.1 we have $p R=p_{i} R$. It follows from Lemma 3.1(2) that $p_{i} p_{j} R=p_{j} p_{i} R$ for all $i$ and $j$. Therefore without loss of generality we may suppose that $p R=p_{1} R$. Thus $I=p I=p_{1} I$ and $p_{1} p_{2} \ldots p_{n} \in p_{1} I$. Hence $p_{2} p_{3} \ldots p_{n} \in I$, which contradicts the minimality of $n$.

(3) Let $c \in C(p R)$ and suppose that $c \in C\left(p^{k} R\right)$ for some positive integer $k$. We must show that $c \in C\left(p^{k+1} R\right)$. Let $x \in R$ with $c x \in p^{k+1} R$. Then $c x \in p^{k} R$ so that $x \in p^{k} R$. Thus 
$x=y p^{k}$ for some $y \in R$. Also $c x=z p^{k+1}$ for some $z \in R$. Hence $c y p^{k}=z p^{k+1}$, and so $c y=z p$. But $c \in C(p R)$. Therefore $y \in p R$ and hence $x \in p^{k+1} R$ as required.

(4) This follows easily from (2) and (3).

(5) Let $x$ be a normal element of $R$ with $x \notin p R$. Then $x \in C(p R)$. Hence $x \in C\left(p^{n} R\right)$ for every positive integer $n$, by (3). Suppose that $x a=p^{n} b$ for some $a, b \in R$ for and positive integer $n$. Because $x \in C\left(p^{n} R\right)$ it follows that $a \in p^{n} R$. Hence $x a \in x p^{n} R$ and the result follows easily.

Let $R$ be a U.F.R. and let $p$ be a prime element of $R$. Then Theorem 3.3 shows that $p R$ satisfies most of the properties which one might expect. The one obvious exception is localisability, and there is no hope of proving this because we did not know enough about the ring $R / p R$. We will show in Section 4 that $p R$ is localisable if $R$ satisfies a polynomial identity, and the Noetherian case was dealt with in the proof of Theorem 2.1 of [6].

The next two results establish the main properties of normal elements and principal ideals in U.F.R.s.

Lemma 3.4. Let $R$ be a U.F.R. and let $x$ be a non-zero normal element of $R$.

(1) If there is no prime element $p$ of $R$ such that $x \in p R$, then $x$ is a unit of $R$.

(2) $x$ is a product of prime elements of $R$.

(3) There are non-associate prime elements $p_{1}, \ldots, p_{n}$ of $R$ and non-negative integers $a(1), \ldots, a(n)$ such that

$$
x R=p_{1}^{a(1)} p_{2}^{a(2)} \ldots p_{n}^{a(n)} R=p_{1}^{a(1)} R \cap \ldots \cap p_{n}^{a(n)} R
$$

(4) Let $P$ be a prime ideal of $R$ which is minimal over $x$. Then height $(P)=1$ and $P=p R$ for some prime element $p$ of $R$.

Proof. (1) Suppose that there is no prime element $p$ of $R$ such that $x \in p R$. By Lemma 3.2 there are prime elements $p_{1}, \ldots, p_{n}$ of $R$ such that $p_{1} p_{2} \ldots p_{n}=x r$ for some $r \in R$. Thus $x r \in p_{n} R$. Also $x \notin p_{n} R$ so that $x \in C\left(p_{n} R\right)$. Therefore $r=s p_{n}$ for some $s \in R$. We have $p_{1} p_{2} \ldots p_{n}=x r=x s p_{n}$ so that $p_{1} p_{2} \ldots p_{n-1}=x s$. Repeating this process gives $1=x t$ for some $t \in R$.

(2) For each prime element $p$ of $R$ there is a positive integer $n$ such that $x \notin p^{n} R$, by Theorem 3.3(2). By Lemma 3.2(2) there are only finitely-many non-associate prime elements $p$ of $R$ such that $x \in p R$. From these two statements it follows that there are (not necessarily distinct) prime elements $p_{1}, \ldots, p_{n}$ of $R$ such that $x=p_{1} p_{2} \ldots p_{n} y$, where $y$ is an element of $R$ with the property that there is no prime element $p$ of $R$ with $y \in p R$. Because $x$ and the $p_{i}$ are normal elements of $R$, so also is $y$. Therefore $y$ is a unit of $R$ by (1), and $x$ is the product of the prime elements $p_{1}, \ldots, p_{n-1}, p_{n} y$.

(3) By (2) and Lemma 3.1(2) there are non-associate prime elements $p_{1}, \ldots, p_{n}$ of $R$ and non-negative integers $a(i)$ such that $x R=p_{1}^{a(1)} p_{2}^{a(2)} \ldots p_{n}^{a(n)} R$. Set $y=p_{2}^{a(2)} p_{3}^{a(3)} \ldots p_{n}^{a(n)}$. 
Then $y \notin p_{1} R$. It follows from Theorem 3.3(5) and Lemma 3.1(2) that $p_{1}^{a(1)} R \cap y R=$ $p_{1}^{a(1)} y R=x R$. We now repeat the process for $y R$, and so on.

(4) Let $P$ be a prime ideal of $R$ which is minimal over $x$. By (2), $x=p_{1} p_{2} \ldots p_{n}$ for some prime elements $p_{i}$ of $R$. For some $i$ we have $p_{i} \in P$. Hence $x R \subseteq p_{i} R \subseteq P$. Therefore $P=p_{i} R$.

Theorem 3.5. Let $R$ be a U.F.R. Then the set of principal ideals of $R$ is closed under finite intersections and satisfies the ascending chain condition.

Proof. Let $x$ and $y$ be non-zero normal elements of $R$. We shall show that $x R \cap y R=z R$ for some normal element $z$ of $R$, and it will follow that the set of principal ideals of $R$ is closed under finite intersections. By Lemma 3.4(3) there are non-associate prime elements $p_{1}, \ldots, p_{n}$ of $R$ and non-negative integers $a(i), b(i)$ such that $x R=$ $p_{1}^{a(1)} R \cap \ldots \cap p_{n}^{a(n)} R$ and $y R=p_{1}^{b(1)} R \cap \ldots \cap p_{n}^{b(n)} R$. For each $i$ set $c(i)=\operatorname{Max}(a(i), b(i))$. Then $x R \cap y R=p_{1}^{c(1)} R \cap \ldots \cap p_{n}^{c(n)} R$. Set $z=p_{1}^{c(1)} p_{2}^{c(2)} \ldots p_{n}^{c(n)}$. Then $x R \cap y R=z R$ by Lemma 3.1(2).

In order to show that $R$ satisfies the ascending chain condition for principal ideals, we let $x$ be a non-zero normal element of $R$ and will show that in fact there are only finitely-many principal ideals of $R$ which contain $x$. Let $y$ be a normal element of $R$ such that $x R \subseteq y R$. By Lemma 3.4(3) there are non-associate prime elements $p_{1}, \ldots, p_{n}$ of $R$ and non-negative integers $a(i)$ such that $x R=p_{1}^{a(1)} R \cap \ldots \cap p_{n}^{a(n)} R$. Similarly $y R=$ $q_{1}^{b(1)} R \cap \ldots \cap q_{k}^{b(k)} R$ for some non-associate prime elements $q_{1}, \ldots, q_{k}$ and non-negative integers $b(j)$. It is now routine to show that for each $j$ there is an $i$ such that $q_{j} R=p_{i} R$ and that $b(j) \leqq a(i)$.

In the commutative case the converse of Theorem 3.5 is also true, but in the non-commutative case a prime ring need not be a U.F.R. even if its set of principal ideals is closed under finite intersections and satisfies the ascending chain condition (see [11, 8 lines up from the bottom of p. 111]).

We next turn to polynomials over a U.F.R. The case of one indeterminate will be of importance in Section 4 as well as being the main step towards dealing with an arbitrary number of indeterminates.

Proposition 3.6. Let $R$ be a U.F.R. and let $X$ be an indeterminate which commutes with the elements of $R$. Then $R[X]$ is a U.F.R.

Proof. As far as possible we will follow the proof of the Noetherian case given in Theorem 3.1 of [6]. The idea is to make use of a simple partial quotient ring $S$ of $R$ and the fact that every ideal of $S[X]$ has a single central generator, in the same sort of way that one can use $\mathbb{Q}[X]$ to prove that $\mathbb{Z}[X]$ is a U.F.D. (where $\mathbb{Q}$ and $\mathbb{Z}$ denote the field of rational numbers and the ring of integers respectively).

Let $D$ be the multiplicative semigroup with 1 generated by the prime elements of $R$. Because the elements of $D$ are normal, we can form a partial quotient ring $S$ of $R$ by inverting the elements of $D$. By Lemma 3.2 we know that every non-zero ideal of $R$ contains an element of $D$, from which it follows that $S$ is a simple ring. To simplify the 
notation we set $R^{*}=R[X]$ and $S^{*}=S[X]$. Because $S$ is simple, every ideal of $S^{*}$ has a single central generator (for a proof of this well-known fact see for example [6, proof of Theorem 3.1]). We shall use this property of $S^{*}$, together with the fact that $S^{*}$ is the partial quotient ring of $R^{*}$ formed by inverting the elements of $D$.

Let $P$ be a non-zero prime ideal of $R^{*}$. We must show that $P$ contains a prime element of $R^{*}$. The prime elements of $R$ are also prime as elements of $R^{*}$, and the elements of $D$ are normal elements of $R^{*}$. If $P \cap D \neq \phi$ then $P$ contains a product of prime elements of $R^{*}$ and hence contains a prime element of $R^{*}$.

From now on we suppose that $P \cap D=\phi$. Then $D \subseteq C(P)$. We wish to show that $P S^{*}$ is a two-sided ideal of $S^{*}$, that is, that $S^{*} P \subseteq P S^{*}$. This would be standard in the Noetherian case, but here and in similar places in Section 4 we must give a direct proof using the special features of the situation. Let $d \in D$ and $x \in P$. It is enough to show that $d^{-1} x \in P S^{*}$. Because $d$ is a normal element of $R^{*}$ we have $x d=d y$ for some $y \in R^{*}$. Thus $d y \in P$ with $d \in C(P)$. Therefore $y \in P$ and $d^{-1} x=y d^{-1} \in P S^{*}$.

Therefore $P S^{*}$ is a two-sided ideal of $S^{*}$. By the discussion above, $P S^{*}=z S^{*}$ for some central element $z$ of $S^{*}$. Also $z=f d^{-1}$ for some $f \in P$ and $d \in D$. Because $d$ is a normal element of $R^{*}$ and $z$ is a central element of $S^{*}, f R^{*}=z d R^{*}=R^{*} z d=R^{*} f$. Let $r$ be a non-zero coefficient of $f$ when $f$ is considered as a polynomial in $X$. There are prime elements $p_{1}, \ldots, p_{n}$ of $R$ such that $r=r^{\prime} p_{1} p_{2} \ldots p_{n}$, for some $r^{\prime} \in R$ with the property that there is no prime element $p$ of $R$ with $r^{\prime} \in p R$ (this follows from Lemma 3.2(2) and Theorem 3.3(2)). Because $r$ is one of the coefficients of $f$, we can conclude that if $p$ is a prime element of $R$ with $f \in p R^{*}$ then $p$ is an associate of $p_{i}$ for some $i$. Therefore there are prime elements $u_{1}, \ldots, u_{t}$ of $R$ such that $f=g u_{1} u_{2} \ldots u_{t}$, for some $g \in R^{*}$ with the property that there is no prime element $p$ of $R$ with $g \in p R^{*}$. Because $f$ and the $u_{i}$ are normal elements of $R^{*}$, so also is $g$. For all $i$ we have $u_{i} \in C(P)$. Also $g u_{1} u_{2} \ldots u_{t} \in P$. Therefore $g \in P$.

Thus $P$ contains a normal element of $g$ of $R^{*}$ with the property that there is no prime element $p$ of $R$ with $g \in p R^{*}$. We shall complete the proof by showing that $P=g R^{*}$ and by symmetry $P=R^{*} g$. Let $w \in P$. We have $P S^{*}=z S^{*}=f d^{-1} S^{*}=f S^{*}=g u_{1} u_{2} \ldots u_{t} S^{*}=$ $g S^{*}$. Because $w \in g S^{*}$ there are prime elements $q_{1}, \ldots, q_{k}$ of $R$ such that $w q_{1} q_{2} \ldots q_{k}=g b$ for some $b \in R^{*}$. But $g \notin q_{k} R^{*}$, so that $g \in C\left(q_{k} R^{*}\right)$. Also $g b \in q_{k} R^{*}$. Hence $b=c q_{k}$ for some $c \in R^{*}$. Therefore $w q_{1} q_{2} \ldots q_{k-1}=g c$. By continuing in this way we eventually obtain $w \in g R^{*}$.

Theorem 3.7. Let $R$ be a U.F.R. and let $W$ be a non-empty set of central indeterminates. Then $R[W]$ is a U.F.R.

Proof. Set $R^{*}=R[W]$ and let $P$ be a non-zero prime ideal of $R^{*}$. There are finitely-many elements $X_{1}, \ldots, X_{n}$ of $W$ such that $P \cap T \neq 0$, where $T=R\left[X_{1}, \ldots, X_{n}\right]$. It is routine to show that $P \cap T$ is a prime ideal of $T$. Also $T$ is a U.F.R., by repeated use of Proposition 3.6. Therefore $P \cap T$ contains a prime element $p$ of $T$. Thus $p \in P$, and $p$ is also prime as an element of $R^{*}$.

We conclude the section with some general information about U.F.D.s which will be used in a special case in Section 4. 
Theorem 3.8. Let $R$ be a U.F.D. in which the elements of $C$, as defined in 2.1, are units. Then every one-sided ideal of $R$ is two-sided. If $R$ is not commutative then every one-sided ideal of $R$ is two-sided and principal (this was proved in the Noetherian case by Gilchrist and Smith in [9]).

Proof. Let $x \in R$. We shall show that $R x R=x R$. From this it will follow that every right ideal of $R$ is two-sided. By Lemma 3.2(2) and Theorem 3.3(2) there are prime elements $p_{1}, \ldots, p_{n}$ of $R$ such that $x=p_{1} p_{2} \ldots p_{n} c$ for some $c \in C$. Because $c$ is a unit of $R$ we have $R x R=R p_{1} p_{2} \ldots p_{n} R=p_{1} p_{2} \ldots p_{n} R=x R$.

Suppose now that $R$ is not commutative and let $P$ be a non-zero prime ideal of $R$. Exactly as in [9] it can be shown that height $(P)=1$. Hence $P=p R$ for some prime element $p$ of $R$. Thus every non-zero prime ideal of $R$ is principal and maximal, and every one-sided ideal of $R$ is two-sided. It follows easily that every non-zero one-sided ideal of $R$ is of the form $q_{1} q_{2} \ldots q_{k} R$ for some prime elements $q_{i}$ of $R$.

\section{Unique factorisation rings which satisfy a polynomial identity}

We shall now specialise to the case of U.F.R.s which satisfy a P.I. (polynomial identity). One reason why these are easier to study compared with general U.F.R.s is that every prime factor ring of a P.I. ring is a Goldie ring. Also prime P.I. rings have a rich supply of central elements. The class of U.F.R.s which satisfy a P.I. includes commutative U.F.D.s; trace rings of generic matrix rings ([13]); polynomials in an arbitrary number of central indeterminates over a finite-dimensional division algebra (Theorem 3.7); the ring of $n$ by $n$ matrices over a commutative Dedekind domain of finite class number $n$; and the group ring $R G$ where $R$ is any U.F.R. which satisfies a P.I. and $G$ is a torsion-free Abelian group which satisfies the ascending chain condition for cyclic subgroups (Example 4.1). But every U.F.D. which satisfies a P.I. is either commutative or is a principal right and left ideal domain (Corollary 4.7).

Let $R$ be a U.F.R. which satisfies a P.I. We shall show that $R / x R$ has an Artinian quotient ring for every normal element $x$ of $R$ (Corollary 4.5); that the height-1 prime ideals of $R$ are localisable and that $R$ is the intersection of the corresponding rings $R_{P}$ (Theorem 4.6); and that $R$ is a maximal order. We shall however, express things in such a way that no knowledge of maximal orders is required. We shall also prove and use the fact that $R$ is the intersection of its simple Artinian quotient ring with a left and right Noetherian principal ideal ring which arises as a partial quotient ring of $R[X]$ (Theorem 4.13). Consequences of this include that $R$ satisfies the ascending chain condition for principal essential right ideals (Corollary 4.14) and every invertible ideal of $R$ is principal (Corollary 4.17).

Some of the results of this section will be proved for U.F.R.s which are left and right Noetherian or which satisfy a P.I.

Example 4.1. Let $R$ be any U.F.R. which satisfies a P.I., and let $G$ be a torsion-free Abelian group which satisfies the ascending chain condition for cyclic subgroups. Then the group ring $R G$ is a U.F.R. which satisfies a P.I. ([7, Theorem 4.2]). The class of 
such groups $G$ is closed under arbitrary direct products and subgroups, so that $G$ could for example be any subgroup of the direct product of an arbitrary number of copies of the infinite cyclic group. The necessity of the condition that $G$ should satisfy the ascending chain condition for cyclic subgroups follows from Theorem 3.5 and the fact that cyclic subgroups of $G$ give rise to principal ideals of $R G$.

Question 4.2. Let $R$ be a ring and let $G$ be a group such that the group ring $R G$ is a prime P.I. ring, i.e. let $R$ be a prime P.I. ring and let $G$ be an Abelian-by-finite group with no proper finite normal subgroups. Is it true that $R G$ is U.F.R. if and only if $G$ satisfies the ascending chain conditions for cyclic subgroups and is dihedral-free (see [1]), and $R$ is a U.F.R.?

The first few results establish some general properties of U.F.R.s which satisfy a P.I., for example localisability at height-1 primes, and the appropriate non-commutative generalisation of the fact that every commutative U.F.D. is integrally-closed.

Proposition 4.3. Let $R$ be a U.F.R. which satisfies a P.I. Suppose that, up to associates, $R$ has only finitely-many prime elements $p_{1}, \ldots, p_{n}$. Then $R$ is a left and right Noetherian semi-local ring with maximal ideals $p_{1} R, \ldots, p_{n} R$. Also every proper two-sided ideal of $R$ is principal and is a product of maximal ideals.

Proof. Let $c \in C\left(p_{1} R\right) \cap \ldots \cap C\left(p_{n} R\right)$. We shall show that $c$ is a unit of $R$. By Theorem 3.3(4) we know that $c$ is a regular element of $R$. Because $R$ is a P.I. ring there is a non-zero central element $z$ of $R$ such that $z=c x$ for some $x \in R$. By Lemma 3.4(2), $z=q_{1} q_{2} \ldots q_{k}$ for some prime elements $q_{i}$ of $R$. But for each $i$ we have $q_{i} R=p_{j} R$ for some $j$. Hence $c \in C\left(q_{i} R\right)$ for all $i$. In particular $c \in C\left(q_{k} R\right)$, and $c x=q_{1} q_{2} \ldots q_{k} \in q_{k} R$. Hence $x=y q_{k}$ for some $y \in R$. Therefore $c y=q_{1} q_{2} \ldots q_{k-1}$. By continuing in this way we find that $c$ is a unit of $R$.

Now let $I$ be any ideal of $R$ which is not contained in any of the ideals $p_{i} R$. We shall show that $I=R$. For each $i$, the ideal $\left(I+p_{i} R\right) / p_{i} R$ of the prime Goldie ring $R / p_{i} R$ is non-zero and so contains a regular element, by Goldie's theorem. Therefore In $C\left(p_{i} R\right) \neq \phi$. Hence there is an element $c$ of $I$ such that $c \in C\left(p_{i} R\right)$ for all $i([17$, Proposition 2.4]). We showed above that $c$ is a unit. Therefore $I=R$. It follows that the $p_{i} R$ are the maximal ideals of $R$. We can now show as in the proof of Theorem 3.8 that every proper ideal of $R$ is a product of ideals of the form $p_{i} R$. Thus every ideal of $R$ is principal, and so $R$ satisfies the ascending chain condition for ideals. It follows by Cauchon's theorem that $R$ is left and right Noetherian ([2, Theorème 1.4]).

Theorem 4.4. Let $R$ be a U.F.R. which satisfies $a$ P.I. and let $p_{1}, \ldots, p_{n}$ be finitely-many non-associate prime elements of $R$. Set $D=C\left(p_{1} R\right) \cap \ldots \cap C\left(p_{n} R\right)$ and let $W$ be the multiplicative semigroup with 1 generated by the prime elements $q$ of $R$ such that $q$ is not an associate of any $p_{i}$. Then $D$ is an Ore subset of $R$. The corresponding partial quotient ring $R_{D}$ is a left and right Noetherian semi-local U.F.R. in which, up to associates, the $p_{i}$ are the only prime elements. Also $R_{D}$ can be formed from $R$ by inverting the elements of $W$. 
Proof. The elements of $W$ are normal in $R$. Hence we can form the corresponding partial quotient ring $T=R_{W}$ of $R$ by inverting the elements of $W$. We shall show that $T$ satisfies the hypotheses of Proposition 4.3. It will then follow easily that the elements of $D$ are units of $T$, that $T=R_{D}$, and that $R_{D}$ has the stated properties.

Because $T$ is a partial quotient ring of $R$ and is a subring of the simple Artinian quotient ring of $R$, it is routine to show that $T$ is a prime P.I. ring. Let $p$ be one of the $p_{i}$. We shall show that $p$ is prime as an element of $T$. Let $w \in W$. By Lemma 3.1(2) we have $p w R=w p R$. Also $w T=T$ by definition of $T$. Hence $p T=p w T=p w R T=w p R T=w p T$, and so $w^{-1} p T=p T$. Hence $T p T=p T$, and by symmetry $p T=T p$. Also because $W \subseteq$ $C(p R)$ it is easy to show that $R \cap p T=p R$. Therefore $p T$ is an ideal of $T$ and is prime.

Let $P$ be a non-zero prime ideal of $T$. Then $P \cap R$ is a non-zero ideal of $R$. By Lemma 3.2(1) there are prime elements $q_{1}, \ldots, q_{s}$ of $R$ such that $q_{1} q_{2} \ldots q_{s} \in P \cap R$. For each $i$, either $q_{i} R=p_{j} R$ for some $j$, or $q_{i} \in W$. Thus each $q_{i}$ is either an associate of one of the prime elements. $p_{j}$ of $T$, or $q_{i}$ is a unit of $T$. Because $q_{1} q_{2} \ldots q_{s} \in P$ it follows that $p_{j} \in P$ for some $j$. Because each $p_{j}$ is prime as an element of $T$ it follows that $T$ is a U.F.R. and that, up to asssociates, the $p_{j}$ are the only prime elements of $T$. Thus $T$ satisfies the hypotheses of Proposition 4.3, as required.

Corollary 4.5. Let $R$ be a U.F.R. which satisfies a P.I. and let $x$ be a non-zero normal element of $R$ which is not a unit. Then $R / x R$ has an Artinian quotient ring. In fact the quotient ring of $R / x R$ is a factor ring of a ring of the form $R_{D}$ as described in Theorem 4.4.

Proof. By Lemma 3.4(3) there are non-associate prime elements $p_{1}, \ldots, p_{n}$ of $R$ and positive integers $a(i)$ such that $x R=p_{1}^{a(1)} R \cap \ldots \cap p_{n}^{a(n)} R$. Set $D=C\left(p_{1} R\right) \cap \ldots \cap C\left(p_{n} R\right)$. Then $D$ is an Ore subset of $R$ by Theorem 4.4, and $D \cong C(x R)$ by Theorem 3.3(3).

Set $S=R_{D}$. We shall show that $x S=S x$. It is then routine to show that the Artinian ring $S / x S$ is the quotient ring of $R / x R$. By Theorem 4.4 we know that $S$ can be formed from $R$ by inverting those prime elements $q$ of $R$ such that $q$ is not an associate of any $p_{i}$. For such $q$ we have $q \in D$ so that $q \in C(x R)$. Also $q R=R q$ so that $x q=q y$ for some $y \in R$. Thus $q y \in x R$ with $q \in C(x R)$. Therefore $y \in x R$ and $q^{-1} x=y q^{-1} \in x S$. It follows that $S x \cong x S$ and by symmetry that $S x=x S$.

Theorem 4.6. Let $R$ be a U.F.R. which satisfies a P.I. and let $P$ be a height-1 prime ideal of $R$. Then the classical localisation $R_{P}$ of $R$ at $P$ exists and is a left and right Noetherian local ring in which every two-sided ideal is principal. Also $R=\cap R_{P}$ as $P$ ranges over the height-1 primes of $R$.

Proof. By Theorem 4.4 we know that $R_{P}$ exists and has the stated properties. Set $T=\cap R_{P}$ as $P$ ranges over the height-1 primes of $R$, and let $S$ be the partial quotient ring of $R$ formed by inverting the prime elements of $R$. Then $S$ is a simple ring, by Lemma 3.2(1). Also $S$ is a P.I. ring, because it is a subring of the classical quotient ring $Q(R)$ of $R$. Therefore $S$ is simple Artinian and $S=Q(R)$. Thus $T=S \cap T$. It is now easy to modify the proof of Theorem 2.10 of [5] to show that $R=S \cap T=T$. 
Corollary 4.7. Let $R$ be a U.F.D. which satisfies a P.I. and suppose that $R$ is not commutative. Then $R$ is a principal right and left ideal domain.

Proof. Let $C$ be as in 2.1. By Theorem 4.6 the elements of $C$ are units of $R$, because each element of $C$ has an inverse in $R_{P}$ for every height-1 prime $P$ of $R$. The result now follows immediately from Theorem 3.8

The next result could be stated in the form "Every U.F.R. which satisfies a P.I. is a maximal order", but for the benefit of the reader who is not familiar with maximal orders we will spell it out in detail. The significance of Corollary 4.8 is that it generalises the fact that every commutative U.F.D. is integrally-closed. Further information about maximal orders can be found in [14].

Corollary 4.8. Let $R$ be a U.F.R. which satisfies a P.I. and suppose that $Q$ is the classical quotient ring of $R$. Let $I$ be a non-zero ideal of $R$. If $q$ is an element of $Q$ such that either $q I \subseteq I$ or $I q \subseteq I$, then $q \in R$.

Proof. Suppose that $q I \subseteq I$. By Lemma 3.2(2) and 3.2(3), there are (not necessarily distinct) prime elements $p_{1}, \ldots, p_{n}$ of $R$ such that $I=K p_{1} \ldots p_{n}$, where $K$ is an ideal of $R$ which is not contained in $p R$ for any prime element $p$ of $R$. Because $q I \subseteq I$ we have $q K \subseteq K$. Let $P$ be a height-1 prime ideal of $R$. Then $K \nsubseteq P$, so that $K \cap C(P) \neq \phi$ by Goldie's theorem applied to the ring $R / P$. Let $R_{P}$ be as in Theorem 4.6. Since $q K \subseteq K$ and $K R_{P}=R_{P}$, we conclude that $q \in R_{P}$ for all such $P$. Therefore $q \in R$ by Theorem 4.6.

Example 4.9. Let $R$ be a U.F.R. which satisfies a P.I. Then $R$ need not be a finite module over its centre even if $R$ is Noetherian. Let $S$ be a Nagarajan's example of a commutative Noetherian U.F.D. which has an automorphism $a$ of order 2 such that the fixed ring $T$ of $a$ is not Noetherian (see for example [10, Section 41, Exercise 4]). Take $R=S[X ; a]$ where multiplication in $R$ is governed by the rule that $X s=a(s) X$ for all $s \in S$. Then $R$ is a U.F.R. ([6, Corollary 4.3]). Also $R$ is a Noetherian P.I. ring. Let $A$ be the centre of $R$. If $R$ were a finite $A$-module then $A$ would be Noetherian ([8, Theorem 3]). But $A=T\left[X^{2}\right]$ which is not Noetherian.

Lemma 4.10. Let $R$ be a U.F.R. which is left and right Noetherian or which satisfies a $P . I .$, and let $c$ be a regular element of $R$. Then, up to associates, there are only finitelymany prime elements $p$ of $R$ such that $c \notin C(p R)$.

Proof. If $R$ is left and right Noetherian then the result follows immediately from Corollary 4.8 and [3, Lemma 3.5]. Now suppose that $R$ satisfies a P.I. Then there is a non-zero central element $z$ of $R$ such that $z \in c R$. Let $p$ be a prime element of $R$ such that $c \notin C(p R)$. Then $(c R+p R) / p R$ is not an essential right ideal of $R / p R$, by Goldie's theorem. Hence $(c R+p R) / p R$ does not contain a non-zero ideal of $R / p R$. But 
$(z R+p R) / p R \subseteq(c R+p R) / p R$. Hence $(z R+p R) / p R=0$, and so $z \in p R$. Up to associates there are only finitely-many such $p$, by Lemma 3.2(2).

The Noetherian case of the next result was part of the second author's Ph.D. thesis at the University of Leeds. He would like to thank Professor J. T. Stafford for suggesting this problem and for improving the method of proof.

Proposition 4.11. Let $R$ be a U.F.R. which is left and right Noetherian or which satisfies a P.I. Set $R^{*}=R[X], W=\left\{p R^{*}: p\right.$ is a prime element of $\left.R\right\}$, and $D=\bigcap_{P \in W} C(P)$. Let $I$ be a right ideal of $R^{*}$ such that for all $P \in W$ we have $I \cap C(P) \neq \phi$. Then $I \cap D \neq \phi$.

Proof. We first show that $I$ is an essential right ideal of $R^{*}$. Let $P \in W$ and let $a \in I \cap C(P)$. We have $P=p R^{*}$ for some prime element $p$ of $R$. Thus $p$ is a prime element of $R^{*}$, and $R^{*}$ is a U.F.R. It follows from Theorem 3.3(4) that $a$ is a regular element of $R^{*}$. Therefore $I$ is an essential right ideal of $R^{*}$.

By "the anti-leading coefficient of a polynomial $r_{0}+r_{1} X+\cdots+r_{n} X^{n}$ " we mean $r_{i}$ if $r_{i} \neq 0$ and $r_{j}=0$ for all $j<i$. Let $K$ be the right ideal of $R$ which consists of 0 together with the anti-leading coefficients of the non-zero elements of $I$. We shall show that $K$ is an essential right ideal of $R$. Let $L$ be a non-zero right ideal of $R$. Because $I$ is an essential right ideal of $R^{*}$, there is a non-zero element $f(X)$ of $R^{*}$ such that $f(X) \in I \cap L R^{*}$. Now $f(X)=r_{0}+r_{1} X+\cdots+r_{n} X^{n}$ for some non-negative integer $n$ and some $r_{j} \in L$. Let $r_{i}$ be the anti-leading coefficient of $f(X)$. Then $r_{i} \neq 0$ because $f(X) \neq 0$. Also $r_{i} \in K \cap L$. Hence $K \cap L \neq 0$, and $K$ is an essential right ideal of $R$. Therefore $K$ contains a regular element of $R$, by Goldie's theorem.

Thus we can fix an element $a$ of $I$ such that the anti-leading coefficient $a_{r}$ of $a$ is a regular element of $R$. By Lemma 4.10 there are only finitely-many non-associate prime elements $p_{1}, \ldots, p_{u}$ of $R$ such that $a_{r} \notin C\left(p_{i} R\right)$. For each such $i$ set $P_{i}=p_{i} R^{*}$. Then $P_{i} \in W$ for all $i$.

Set $W^{\prime}=W-\left\{P_{1}, \ldots, P_{u}\right\}$. Let $P \in W^{\prime}$. We have $P=p R^{*}$ for some prime element $p$ of $R$ such that $a_{r} \in C(p R)$. Because $a_{r}$ is the anti-leading coefficient of $a$, it follows easily that $a \in C(P)$ for all $P \in W^{\prime}$.

For each $i$ from 1 to $u$ we have $I \cap C\left(P_{i}\right) \neq \phi$. By [17, Proposition 2.4] we can fix $b \in I$ such that $b \in C\left(P_{i}\right)$ for all $i$. For each positive integer $j \geqq r+1$ set $f_{j}=a+b X^{j}$, where the anti-leading term of $a$ is $a_{r} X^{r}$. Then $f_{j} \in I$ for all $j$. We shall complete the proof by showing that $f_{j} \in D$ for some $j$. Because the anti-leading coefficient of $f_{j}$ is $a_{r}, f_{j} \in C(P)$ for all $j$ and all $P \in W^{\prime}=W-\left\{P_{1}, \ldots, P_{u}\right\}$.

Suppose that there is no value of $j$ such that $f_{j} \in D$; we shall obtain a contradiction. Then for each $j$ there is a positive integer $i$ with $1 \leqq i \leqq u$ such that $f_{j} \notin C\left(P_{i}\right)$. We can fix a value of $i$ such that $J=\left\{j: f_{j} \notin C\left(P_{i}\right)\right\}$ is an infinite set. We can enumerate the elements of $J$ as an increasing sequence $j(1)<j(2)<\ldots$. For each $j \in J$ there exists $g_{j} \in R^{*}$ such that $f_{j} g_{j} \in P_{i}$ and $g_{j} \notin P_{i}$. For each positive integer $s$ set $A(s)=g_{j(1)} R^{*}+\cdots+g_{j(s)} R^{*}$. Then the right ideals $\left(A(s)+P_{i}\right) / P_{i}$ form an ascending chain in the prime Goldie ring $R^{*} / P_{i}$. Hence there is a positive integer $s$ such that $\left(A(s)+P_{i}\right) / P_{i}$ is an essential submodule of $\left(A(s+1)+P_{i}\right) / P_{i}$. It follows that $\left(A(s+1)+P_{i}\right) /\left(A(s)+P_{i}\right)$ is torsion as a right 
$R^{*} / P_{i}$-module. In particular there exists $c \in C\left(P_{i}\right)$ such that $\left(g_{j(s+1)}+P_{i}\right) c \in\left(A(s)+P_{i}\right) / P_{i}$, which means that $g_{j(s+1)} c \in A(s)+P_{i}=g_{j(1)} R^{*}+\cdots+g_{j(s)} R^{*}+P_{i}$.

Thus there is a positive integer $t$ minimal with respect to the property that

$$
g_{j(1)} h_{j(1)}+\cdots+g_{j(t)} h_{j(t)} \in P_{i} \text { for some } h_{j(k)} \in R^{*} \text { where } h_{j(t)} \in C\left(P_{i}\right) \text {. }
$$

By our choice of $f_{j}$ and $g_{j}, f_{j} g_{j} \in P_{i}$ for all $j$, and recall that $a=f_{j}-b X^{j}$. By multiplying (1) on the left by $a$, we thus obtain

$$
b g_{j(1)} h_{j(1)} X^{j(1)}+\cdots+b g_{j(t)} h_{j(t)} X^{j(t)} \in P_{i}
$$

But $b \in C\left(P_{i}\right)$. Therefore

$$
g_{j(1)} h_{j(1)} X^{j(1)}+\cdots+g_{j(t)} h_{j(t)} X^{j(t)} \in P_{i}
$$

Suppose that $t \neq 1$. By multiplying (1) by $X^{j(1)}$ and then subtracting the resulting element from (3) we obtain

$$
g_{j(2)} h_{j(2)}\left(X^{j(2)}-X^{j(1)}\right)+\cdots+g_{j(t)} h_{j(t)}\left(X^{j(t)}-X^{j(1)}\right) \in P_{i} .
$$

But $h_{j(t)}\left(X^{j(t)}-X^{j(1)}\right) \in C\left(P_{i}\right)$. Thus (4) contradicts the choice of $t$. Therefore $t=1$, and $g_{j(1)} h_{j(1)} \in P_{i}$ with $h_{j(1)} \in C\left(P_{i}\right)$. But then $g_{j(1)} \in P_{i}$, which is the desired contradiction.

Corollary 4.12. Let $R^{*}$ and $D$ be as in Proposition 4.11. Then $D$ is an Ore subset of $R^{*}$.

Proof. Let $c \in D$ and $a \in R^{*}$. Set $I=\left\{z \in R^{*}: a z \in c R^{*}\right\}$. Then $I$ is a right ideal of $R^{*}$. Let $p$ be a prime element of $R$. Then $R^{*}$ satisfies the Ore condition with respect to $C\left(p R^{*}\right)$ by Proposition 3.6 and Theorem 4.6. Because $c \in C\left(p R^{*}\right)$ it follows that $a z=c b$ for some $b \in R^{*}$ and $z \in C\left(p R^{*}\right)$. Thus $z \in I$ and so $I \cap C\left(p R^{*}\right) \neq \phi$. Therefore $I \cap D \neq \phi$ by Proposition 4.11, i.e. $a d \in c R^{*}$ for some $d \in D$. This establishes the right Ore condition with respect to $D$, and the left Ore condition follows by symmetry.

Theorem 4.13. Let $R$ be a U.F.R. which is left and right Noetherian or which satisfies a P.I. Set $R^{*}=R[X], W=\left\{p R^{*}: p\right.$ is a prime element of $\left.R\right\}$, and $D=\bigcap_{P E W} C(P)$. Let $S$ be the simple ring formed from $R$ by inverting the prime elements of $R$, and let $T$ be the partial quotient ring of $R^{*}$ formed by inverting the elements of $D$. Then $T$ is a left and right Noetherian ring in which every proper ideal is of the form $p_{1} p_{2} \ldots p_{n} T=T p_{1} p_{2} \ldots p_{n}$ for some prime elements $p_{i}$ of $R$. Also $R=S \cap T$ where $S$ and $T$ are regarded in the natural way as being subrings of the quotient ring of $R^{*}$.

Proof. Let $p$ be a prime element of $R$. We shall show that $p T=T p$. Suppose that $d \in D$. By Corollary 4.12 we have $p c=d b$ for some $c \in D$ and $b \in R^{*}$. Thus $d b \in p R^{*}$ with 
$d \in C\left(p R^{*}\right)$. Hence $b \in p R^{*}$. Therefore $d^{-1} p=b c^{-1} \in p T$. It follows that $T p \subseteq p T$ and by symmetry that $T p=p T$.

Let $I$ be an ideal of $T$ such that there is no prime element $p$ of $R$ with $I \subseteq p T$. We shall show that $I=T$, and it will then follow, as in the last paragraph of the proof of Proposition 4.3, that $T$ has the stated properties. Let $p$ be a prime element of $R$. Then $I \nsubseteq p T$, so that $I \cap R^{*} \nsubseteq p R^{*}$ (this is because $I=\left(I \cap R^{*}\right) T$ ). Thus $I \cap R^{*}$ is a two-sided ideal of $R^{*}$ which is not contained in the prime ideal $p R^{*}$. It follows by Goldie's theorem that $I \cap R^{*} \cap C\left(p R^{*}\right) \neq \phi$. By Proposition 4.11 we have $I \cap R^{*} \cap D \neq \phi$, and so $I \cap D \neq \phi$. Thus $I=T$.

Let $s \in S \cap T$. Because $s \in S$ we have $p_{1} p_{2} \ldots p_{n} s \in R$ for some prime elements $p_{i}$ of $R$. Also $s d \in R^{*}$ for some $d \in D$. Thus $p_{1} p_{2} \ldots p_{n} s d \in p_{1} R^{*} \cap R d$. Because $d \in C\left(p_{1} R^{*}\right)$ we have $p_{1} R^{*} \cap R d=p_{1} R^{*} \cap\left(R \cap p_{1} R^{*}\right) d=p_{1} R^{*} \cap p_{1} R d=p_{1} R d$. Hence $p_{1} p_{2} \ldots p_{n} s d \in p_{1} R d$ so that $p_{2} p_{3} \ldots p_{n} s \in R$. By continuing in this way we eventually obtain $s \in R$.

Corollary 4.14. Let $R$ be a U.F.R. which satisfies a P.I. Then $R$ satisfies the ascending chain condition for right ideals of the form $c R$ where $c$ is a regular element of $R$.

Proof. We shall use the notation of Theorem 4.13. Let $c$ be a regular element of $R$. Because $S$ is the classical quotient ring of $R$ in the P.I. case of Theorem 4.13, we have $c S=S$. Hence $c R=c(S \cap T)=c S \cap c T=S \cap c T$. The result now follows because $T$ is right Noetherian.

Notation 4.15. Let $R$ be a prime Goldie ring with classical quotient ring $Q$ and let $I$ be any subset of $Q$. Set $I_{l}=\{q \in Q: q I \subseteq R\}$ and $I_{r}=\{q \in Q: I q \subseteq R\}$.

In the language of maximal orders, the next result says that any reflexive ideal of a suitable U.F.R. is principal.

Theorem 4.16. Let $R$ be a U.F.R. which is left and right Noetherian or which satisfies a P.I., and let $I$ be an ideal of $R$ such that $\left(I_{l}\right)_{r}=I$ in the notation of 4.15. Then $I$ is principal.

Proof. We may suppose that $0 \neq I \neq R$. By Lemma 3.2(2) and Theorem 3.3(2), there are prime elements $p_{1}, \ldots, p_{n}$ of $R$ such that $I=p_{1} p_{2} \ldots p_{n} K$, where $K$ is an ideal of $R$ such that there is no prime element $p$ of $R$ with $K \subseteq p R$. Set $x=p_{1} p_{2} \ldots p_{n}$.

We have $I_{l} x K=I_{l} I \subseteq R$ so that $I_{l} x \subseteq K_{l}$. Hence $I_{l} x\left(K_{l}\right)_{r} \subseteq R$, and so $x\left(K_{l}\right)_{r} \subseteq\left(I_{l}\right)_{r}=I=$ $x K$. Therefore $\left(K_{l}\right)_{r} \subseteq K$ so that $\left(K_{l}\right)_{r}=K$. In the notation of Theorem 4.13 we have $K_{l}(K S \cap K T) \subseteq K_{l} K S \cap K_{l} K T \subseteq R S \cap R T=S \cap T=R$. Hence $K S \cap K T \subseteq\left(K_{l}\right)_{r}=K$, and it follows that $K S \cap K T=K$. By Lemma 3.2(1) we know that $K$ contains a product of prime elements of $R$. Hence $K S=S$. For each prime element $p$ of $R$ we have $K \nsubseteq p R$ so that $K \nsubseteq p R^{*}$. Thus $K R^{*}$ is a two-sided ideal of $R^{*}$ with $K R^{*} \nsubseteq p R^{*}$. Therefore $K R^{*} \cap C\left(p R^{*}\right) \neq \phi$. By Proposition 4.11 we have $K R^{*} \cap D \neq \phi$, so that $T=K R^{*} T=K T$. Hence $K=K S \cap K T=S \cap T=R$. 
Corollary 4.17. Let $R$ be a U.F.R. which is left and right Noetherian or which satisfies $a$ P.I., and let $I$ be an invertible ideal of $R$. Then $I$ is principal.

Proof. To say that $I$ is invertible means that $I X=X I=R$ for some $R$-subbimodule $X$ of the quotient ring of $R$. In the notation of 4.15 we have $X \subseteq I_{1}$. Also $I_{1}=I_{l} R=$ $I_{l} I X \subseteq R X=X$. Therefore $I_{l}=X$. Hence $\left(I_{l}\right)_{r}=X_{r}=R X_{r}=I X X_{r} \subseteq I$. It follows that $\left(I_{l}\right)_{r}=I$ and Theorem 4.16 applies.

Our final aim is to show that if $R$ is a U.F.R. which satisfies a P.I. then $R$ is a prime Krull ring as studied in [18].

Definition 4.18. Let $R$ be a prime Goldie ring with classical quotient ring $Q$ and let $I$ be a right ideal of $R$. We say that $I$ is closed if $I=\{x \in R: x K \cong I$ for some essential right ideal $K$ of $R$ with $K_{l}=R$ in the notation of 4.15$\}$.

Theorem 4.19. Let $R$ be a U.F.R. which satisfies a P.I. Then $R$ satisfies the ascending chain condition for closed right ideals.

Proof. We shall use the notation of Theorem 4.13. Let $A$ be a closed right ideal of $R$ and set $B=A S \cap A T$. Note that $B \subseteq R$ by Theorem 4.13. We shall show that $B=A$, and the result will then follow easily because both $S$ and $T$ are right Noetherian rings (in fact $S$ is the simple Artinian quotient ring of $R$ ).

Let $b \in B$ and set $K=\{r \in R: b r \in A\}$. Because $b \in A S$ we have $b c \in A$ for some regular element $c$ of $R$ (we can take $c$ to be a product of prime elements of $R$ ). Thus $c \in K$ and so $K$ is an essential right ideal of $R$. We shall show that $K_{l}=R$, and it will follow that $b \in A$ because $A$ is closed. Because $b \in A T$ we have $b d \in A R^{*}$ for some $d \in D$. Now $d=r_{0}+r_{1} X+\cdots+r_{n} X^{n}$ for some $r_{i} \in R$. Thus $b r_{0}+b r_{1} X+\cdots+b r_{n} X^{n} \in A R^{*}$, so that $b r_{i} \in A$ for all $i$. Thus $r_{i} \in K$ for all $i$. Hence $K_{l} r_{i} \subseteq R$ for all $i$, and so $K_{l} d \subseteq R^{*}$. Therefore $K_{l} \subseteq T$. But $K_{l} \subseteq S$ because $S$ is the classical quotient ring of $R$. Hence $K_{l} \subseteq R$ by Theorem 4.13, and clearly $R \cong K_{l}$. Therefore $K_{l}=R$ as required.

Corollary 4.20. Let $R$ be a U.F.R. which satisfies a P.I. Then $R$ is a prime Krull ring as studied in [18].

Proof. By definition, a prime Krull ring is a prime Goldie ring which satisfies the conclusions of Corollary 4.8 and Theorem 4.19.

Acknowledgement. The third author wishes to thank his Ph.D. supervisor, Dr C. R. Hajarnavis of the University of Warwick, for his help and encouragement.

\section{REFERENCES}

1. K. A. Brown, Height one primes of polycyclic group rings, J. London Math. Soc. (2) 32 (1985), 426-438. 
2. G. CAUChon, Anneaux semipremiers Noetheriens, a identites polynomiales, Bull. Soc. Math. France 104 (1976), 99-111.

3. M. Chamarie, Localisations dans les ordres maximaux, Comm. Algebra 2 (1974), 279-293.

4. A. W. Chatters and C. R. Hajarnavis, Rings with chain conditions (Pitman, London, 1980).

5. A. W. Chatrers, Non-commutative unique factorisation domains, Math. Proc. Cambridge Philos. Soc. 95 (1984), 49-54.

6. A. W. Chatters and D. A. Jordan, Non-commutative unique factorisation rings, J. London Math. Soc. (2) 33 (1986), 22-32.

7. A. W. Chatters and J. Clark, Group-rings which are unique factorisation rings, Comm. Algebra, to appear.

8. E. Formanek and A. V. Jategaonkar, Subrings of Noetherian rings, Proc. Amer. Math. Soc. 46 (1974), 181-186.

9. M. P. GILChRIST and M. K. SMITH, Non-commutative UFD's are often PID's, Math. Proc. Cambridge Philos. Soc. 95 (1984), 417-419.

10. R. Gilmer, Multiplicative ideal theory (Marcel Dekker Inc., 1982).

11. D. A. Jordan, Unique factorisation of normal elements in non-commutative rings, Glasgow Math. J. 31 (1989), 103-113.

12. I. KaPLANSKy, Commutative rings, revised edition (U. of Chicago Press, 1974).

13. L. le BruYn, Trace rings of generic matrix rings are unique factorisation domains, Glasgow Math. J. 28 (1986), 11-13.

14. G. Maury and J. Raynaud, Ordres maximaux au sens de $K$. Asano (Springer Lecture Notes in Mathematics Vol. 808).

15. J. C. McConnell and J. C. Rosson, Non-commutative Noetherian rings (Wiley, 1987).

16. L. S. Rowen, Polynomial identities in ring theory (Academic Press, 1980).

17. J. T. Stafford, Noetherian full quotient rings, Proc. London Math. Soc. (3) 45 (1982), 385-404.

18. J. T. STAfFord, Modules over prime Krull rings, J. Algebra 95 (1985), 332-342.

SChOol of Mathematics UNIVERSITY OF BRISTOL UNIVERSITY WALK

BRISTOL BS8 1TN
Science and Medical Department Oxford University Press

Walton Street

OXFORD OX2 6DP
Mathematical InSTITUTE UNIVERSITY OF WARWICK Coventry CV4 7AL 